\title{
Identification of atherosclerosis-associated conformational heat shock protein 60 epitopes by phage display and structural alignment
}

\author{
Hannes Perschinka ${ }^{\mathrm{a}, 1}$, Bernd Wellenzohn ${ }^{\mathrm{b}, 2}$, Walther Parson ${ }^{\mathrm{c}}$, Ruurd van der Zee ${ }^{\mathrm{d}}$, \\ Johann Willeit ${ }^{\mathrm{e}}$, Stefan Kiechl ${ }^{\mathrm{e}}$, Georg Wick ${ }^{\mathrm{a}, *}$ \\ ${ }^{a}$ Division of Experimental Pathophysiology and Immunology, Biocenter, Innsbruck Medical University, Fritz-Pregl-Str. 3/IV, 6020 Innsbruck, Austria \\ ${ }^{\mathrm{b}}$ Institute of General, Inorganic and Theoretical Chemistry, University of Innsbruck, Austria \\ ${ }^{\mathrm{c}}$ Institute of Legal Medicine, Innsbruck Medical University, Austria \\ d Department of Infectious Diseases and Immunology, Faculty of Veterinary Medicine, University of Utrecht, The Netherlands \\ e University Clinic for Neurology, Innsbruck Medical University, Austria
}

Received 25 April 2006; received in revised form 22 September 2006; accepted 27 September 2006

\begin{abstract}
Autoimmune reactions to HSP60 are believed to play a key role during development of early atherosclerosis. Due to the high degree of phylogenetic conservation between microbial and human HSP60, bacterial infections might be responsible for inducing cross-reactivity to self HSP60, which is expressed on the surface of arterial endothelial cells stressed by classical atherosclerosis risk factors.

Conformational epitopes recognized by polyclonal anti-mycobacterial HSP60 antibodies from subjects with atherosclerosis were identified using a phage displayed random library of cyclic constrained 7 mer peptides. After five rounds of selection, DNA sequencing of strongly binding clones revealed that one peptide motif (CIGSPSTNC) was present in 64\% of all clones, and a second motif (CSFHYQNRC) in 14\%. Using a newly developed method for structural alignment of small constrained peptides onto a protein surface, we located the motif present in $14 \%$ of all clones on the surface of mycobacterial HSP60. The motif present in $64 \%$ of all clones was found on the surface of mycobacterial HSP60 as well as in the homologous region of human HSP60, which makes this epitope a promising candidate for further investigations on cross-reactive epitopes involved in early atherogenesis.
\end{abstract}

(C) 2006 Elsevier Ireland Ltd. All rights reserved.

Keywords: Aging; Antibodies; Atherosclerosis; Heat shock protein 60; Phage display; Molecular modelling; Structural alignment

\section{Introduction}

During the last decade, the classical theories of atherogenesis have been supplemented by the concept that immunologic-inflammatory processes play an important role at various stages of the disease [1]. However, the initial trig-

\footnotetext{
* Corresponding author. Tel.: +43 5129003 70960; fax: +43512900373960.

E-mail address: Georg.Wick@i-med.ac.at (G. Wick).

${ }^{1}$ Present address: Gebro Pharma GmbH, Research and Development, Fieberbrunn, Austria.

${ }^{2}$ Present address: Boehringer Ingelheim Pharma $\mathrm{GmbH}$, Research and Development, Biberach an der Riß, Germany.
}

gering event and the involved (auto)-antigens still remain controversial.

Heat shock proteins of the $60 \mathrm{kDa}$ family (HSP60) belong to one of the most highly conserved protein families in evolution [2]. HSP60s from different bacterial species have homology higher than $97 \%$ at their protein levels, while prokaryotic and human HSP60 (hHSP60) still show over 70\% sequence homology at most conserved regions. Since microbial HSP60s serve as major antigens in protection from, and pathogenesis of infectious diseases, autoimmune disorders such as rheumatoid arthritis, systemic sclerosis, psoriasis, Kawasaki's disease or Behcet's disease, are thought to be triggered by shared B- and T-cell epitopes cross-reactive between eukaryotic and prokaryotic HSP60 [3]. Interestingly, 
HSP60, which is physiologically active inside mitochondria, was found also on the surface of stressed eukaryotic cells, where it may serve as a danger signal and as target-(auto)antigen for immune reaction [4-6].

Since chronic bacterial infections are known to be a risk factor for atherosclerosis [7-9], immunological crossreactions between bacterial and hHSP60 might be involved in atherogenesis. In a prospective longitudinal study, we showed [10,11], and others confirmed [12,13], that elevated levels of anti-mycobacterial HSP65 (mHSP65) antibodies cross-reacting with hHSP60 serve as a prognostic marker of the incidence, prevalence, severity and progression of carotid atherosclerosis in a clinically healthy population. Recent evidence from other groups confirms the correlation of serum antibody reactivity to hHSP60 with atherosclerosis $[14,15]$.

We previously reported linear epitopes shared between human and microbial HSP60 and recognized by serum antibodies from subjects with atherosclerosis [16]. However, antibodies recognize three-dimensional structural motifs that are not necessarily composed of consecutive linear amino acid sequences. The method of choice to identify these so-called "non-linear" or "conformational" epitopes is the screening of random libraries of phage-displayed, cyclicconstrained, peptides [17]. However, there is no definitive technique for the back-assignment of these cyclic peptide structures onto the surface of the target protein, and most studies have identified the putative epitopes represented by the phage display-derived peptides by visual comparison of three-dimensional structures or by simple linear sequence alignment experiments.

The aim of this study was to identify phage display-derived cyclic peptide structures recognized by serum antibodies to HSP60 isolated from subjects with atherosclerosis, as well as improve assignment of these cyclic peptide structures to specific regions of human and mycobacterial HSP60 by defining a new method for structural alignment of the cyclic peptide structures onto the protein surface.

\section{Material and methods}

\subsection{Purification of serum anti-HSP 60 antibodies}

Blood samples were taken from participants in the Bruneck Study, a large population-based study on atherosclerosis prevention [18]. Antibodies against mHSP65 were determined by ELISA following an established protocol [10]. For purification of anti-HSP antibodies, sera of five subjects with titers $\geq 1: 1280$ and sonographically proven atherosclerotic lesions in the carotid artery were pooled. Affinity chromatography of serum was performed using a previously described method [19]. Briefly, complement of pooled sera was heatinactivated and immunoglobulins were collected by standard ammonium sulphate precipitation. The precipitate was taken up (PBS, pH 7.2), and allowed to run through a chromatog- raphy column loaded with $2 \mathrm{ml}$ agarose gel beads (AffiGel 15, BioRad, Hercules, USA) coupled with $3 \mathrm{mg}$ recombinant mHSP65 (obtained from the Concerted Action "Heat Shock Proteins in Inflammatory Diseases", supported by the European Commission, project BMH4-CT98-3935). After washing away unbound proteins, specific immunoglobulins were recovered by $20 \mathrm{mM}$ hydrochloric acid elution. Fractions containing antibodies were immediately neutralized, pooled, dialyzed against $\mathrm{PBS}, \mathrm{pH} 7.2$ and adjusted to original serum volume. The titers of purified serum anti-HSP antibodies were similar to those of the original serum pool $(\geq 1: 1280)$, whereas unbound immunoglobulins had no measurable HSP antibody titer.

\subsection{Phage display_general biopanning procedure}

All Phage display methods followed manufacturer's instructions (Ph.D.-C7C Phage Display Peptide Library Kit, New England Biolabs, Beverley, USA). Accordingly, the library consisted of $1.2 \times 10^{9}$ sequences (compared to $20^{7}=1.28 \times 10^{9}$ possible 7 -residue sequences, amplified once to yield $\approx 200$ copies of each sequence in the starting material). Petri dishes (Falcon 35-3001) were coated with the respective target overnight at $4{ }^{\circ} \mathrm{C}$ (polyclonal human antimHSP65 1:500 or streptavidin 1:1000, in PBS). After blocking with bovine serum albumin (BSA) $5 \mathrm{mg} / \mathrm{ml}$ ) for $1 \mathrm{~h}$ at room temperature, $2 \times 10^{11}$ phages from the starting library were added and incubated for $1 \mathrm{~h}$ in TBS/Tween $(0.1 \%)$. Phages bearing a sequence motif binding to the antibody adhered to the plate, while unbound phages were removed by extensive washing 10 times with TBS/Tween $(0.1 \%)$. Specifically bound phages were eluted with weak acid for $10 \mathrm{~min}(0.2 \mathrm{M}$ glycine, $\mathrm{pH} 1.1)$ and immediately neutralized with sodium hydroxide $(0.5 \mathrm{M})$. The number of recovered phages was determined by titration (see below). The eluate was immediately added to $20 \mathrm{ml}$ of Escherichia coli strain ER2738 ( $\left.\mathrm{OD}_{600} \sim 0.2\right)$ in LB-medium and amplified for $5 \mathrm{~h}$ at $37^{\circ} \mathrm{C}$ with vigorous shaking. The culture supernatant was then centrifuged twice at $14,000 \times g$ to remove any cells and the phages precipitated over night at $4{ }^{\circ} \mathrm{C}$ by adding $1 / 6$ volume polyethylene glycol (20\%). The next day, the precipitate was centrifuged at $14,000 \times g$ at $4{ }^{\circ} \mathrm{C}$ for $15 \mathrm{~min}$, the resultant pellet suspended in $1 \mathrm{ml}$ TBS and re-centrifuged, and the supernatant transferred to a fresh tube and precipitated with polyethylene glycol $(20 \%)$ for at least $1 \mathrm{~h}$ at $4{ }^{\circ} \mathrm{C}$. After centrifugation $\left(14,000 \times g\right.$ at $4{ }^{\circ} \mathrm{C}$ for $\left.15 \mathrm{~min}\right)$, the pellet was suspended in $200 \mu \mathrm{l}$ TBS/ $0.02 \% \mathrm{NaN}_{3}$ and the number of phages determined by titration (see below). Concentration was adjusted to $10^{10}$ phages/ $\mu$ l and approximately $2 \times 10^{11}$ phages were used again for the next selection cycle. For streptavidin three, for the polyclonal anti-mHSP65 antibody, five selection cycles were performed. Before the last two selection cycles of the polyclonal anti-mHSP65 antibody, a negative selection using a polyclonal nonsense antibody ( $\mathrm{sc}$ 493, Santa Cruz Biotechnology, USA) coating 1:500 in PBS, blocking with BSA $(5 \mathrm{mg} / \mathrm{ml})$ was performed. 


\subsection{Phage display—phage titering}

Since the library phages are derived from the common M13mp19 cloning vector, which carries the lac $\mathrm{Z} \alpha$ gene, phage plaques appear blue when grown on IPTG/XGal plates. To determine phage concentrations, 10 -fold serial dilutions of the phage containing suspension were prepared in LBmedium $\left(10^{1}\right.$ to $10^{4}$ for unamplified panning eluates, $10^{8}$ to $10^{11}$ for amplified cell culture supernatants). Ten microliters of phage dilutions were incubated with $200 \mu$ l of $E$. coli strain ER2738 in LB medium $\left(\mathrm{OD}_{600} \sim 0.5\right)$ for $10 \mathrm{~min}$ at room temperature, suspended in $3 \mathrm{ml}$ of melted agarose at $45^{\circ} \mathrm{C}$ and poured onto a pre-warmed IPTG/XGal plate. After growing for $8 \mathrm{~h}$ at $37^{\circ} \mathrm{C}$, blue plaques were counted on plates having $\sim 10^{2}$ plaques. The concentration of phages was then calculated by the dilution factor of the counted plate.

\subsection{Phage display—DNA isolation and sequencing}

Single clones were picked from plates containing no more than $\sim 10^{2}$ plaques and amplified in $1 \mathrm{ml}$ of ER2738 in $\mathrm{LB}$ medium $\left(\mathrm{OD}_{600} \sim 0.2\right)$ for $5 \mathrm{~h}$ at $37^{\circ} \mathrm{C}$ with vigorous shaking. After centrifugation $\left(14,000 \times g\right.$ at $4{ }^{\circ} \mathrm{C}$ for $1 \mathrm{~min}), 800 \mu \mathrm{l}$ of the supernatant was transferred to a fresh tube and precipitated with $200 \mu$ l polyethylene glycol $(20 \%)$ for $15 \mathrm{~min}$ at $4{ }^{\circ} \mathrm{C}$. Phages were then pelleted by centrifugation, lysed in $100 \mu \mathrm{l} 4 \mathrm{M}$ iodide buffer, and single stranded phage DNA precipitated by incubation with $250 \mu \mathrm{l}$ ethanol for $10 \mathrm{~min}$ and subsequent centrifugation $(14,000 \times g, 10 \mathrm{~min})$. The DNA pellet was washed twice with $70 \%$ ethanol, dried overnight and suspended in $30 \mu \mathrm{l}$ TE-buffer. DNA content was verified by electrophoresis on agarose gels. Automated DNA-sequencing with dye-labeled dideoxyoligonucleotides was performed using the -96 primer (5'-CCCTCATAGTTAGCGTAACG$3^{\prime}$, Mircosynth, Switzerland). The readout sequence corresponded to the anticodon strand of the template. After writing out the complementary strand, the encoded protein sequence was translated.

\subsection{Phage display—ELISA with isolated phage clones}

ELISA plates (PolarPlastic, Eschenbach, Switzerland) were coated with $100 \mu \mathrm{l}$ of the respective substrate solution in PBS overnight at $4{ }^{\circ} \mathrm{C}$ (Streptavidin $5 \mu \mathrm{g} / \mathrm{ml}$, polyclonal human anti-HSP65 1:500). After blocking with $5 \%$ skim milk in PBS for $1 \mathrm{~h}$ at room temperature, $1.5 \times 10^{8}$ phages (in $100 \mu \mathrm{l}$ PBS) were added and incubated for $1 \mathrm{~h}$ at room temperature. Plates were then washed twice with PBS/Tween $(0.05 \%)$, and $100 \mu l$ peroxidase-labeled antiM13-phage antibody was added for another hour. After washing four times with PBS/Tween $(0.05 \%)$, the reaction was visualized with ABTS (Sigma, Munich, Germany) and optical density read at $410 \mathrm{~nm}$ in a Dynatech MR5000 ELISA reader.

\subsection{Phage display-positive control}

The methods used for phage display were validated by repeating a previously published experiment, relying on the binding motif of a phage-displayed-constrained 7 mer random peptide library to streptavidin [20].

\subsection{Peptide structure prediction by simulated annealing}

The cyclic peptide structures (CIGSPSTNC, CSFHYQNRC, CPESHINQC) were generated in silico by simulated annealing in Amber 6.0 [21]. First a solvation (12 water-box in every direction) of the starting structures was performed by energy minimization according to a conjugate gradient method for 1000 steps. The simulation of the structure (simulated annealing procedure) was then performed using the all-atom force field of Cornell [22]: First the temperature of the system was risen from 0 to $800 \mathrm{~K}$ during the first $4 \mathrm{ps}$, then the system was allowed to find into a new energy minimum by cooling down slowly for $25 \mathrm{ps}$. The resulting structure then served as starting structure for the next simulation and three simulations were carried out for each peptide. Comparison of the trajectories showed that each simulation resulted in a new minimum (data not shown).

\subsection{Homology modelling of protein structures}

Since no experimental structural data is available for hHSP60 or mHSP65, respective amino acid sequences were submitted to SwissModel (http://swissmodel.expasy.org/ swissmod/SWISS-MODEL.html) for comparative modelling [23]. The structure of hHSP60 was generated based on PdB-entries of four bacterial HSP60s (1GRL 1OEL, 1AON, $1 \mathrm{JON})$. Due to sequence identity of $51 \%$ and homology of $72 \%$ with the templates (all template structures are from GroEL), reliability of the model should be very high, resulting in a RMSD for backbone atoms $\leq 4 \AA$ [24]. The mHSP65 structure was obtained using $1 \mathrm{GRL}$ and $1 \mathrm{AON}$ as template. Identity of 60 and homology of $88 \%$ represent nearly optimal conditions for homology modelling. Model quality should therefore be comparable to medium resolution NMR or low resolution X-ray structures [25]. Energy minimization and model refinement was performed with GROMOS96 force field [26] (default parameters from SwissModel).

\subsection{Accessible surface area prediction}

Surface-exposed amino acids were calculated by the "GetArea" routine (http://www.scsb.utmb.edu/cgi-bin/get_a_ form.tcl) implemented in the "FANTOM" program [27]. An amino acid " $X$ " was only considered "buried" if the ratio of sidechain surface area to "random coil" value per residue is less than $20 \%$. The "random coil" value of a residue " $X$ " is the average solvent-accessible surface area of " $\mathrm{X}$ " in the tripeptide Gly-X-Gly in an ensemble of 30 random conformations. 


\subsection{Program for structural alignment}

The input file for the protein structure must contain coordinates of surface-exposed $\mathrm{C} \beta$ atoms ( $\mathrm{C} \alpha$ for glycine) and the types of the corresponding amino acids. The number of protein $\mathrm{C} \beta$ atoms is unlimited, the peptide input file must contain five $C \beta s$ and the types of the corresponding amino acids. First, the distances between each $\mathrm{C} \beta$ atom in the protein are calculated and the four nearest atoms for every $C \beta$ are determined. Then, the best superimposition with each permutation of the peptide $C \beta$ coordinates is determined for each set of coordinates $(C \beta+$ four nearest $C \beta)$ according to the Kabsch algorithm $[28,29]$. A score is calculated for every $C \beta$ of a superimposition based on similarity of the superimposed amino acids (according to Tudos' amino acid substitution matrix for isomorphic replacement) [30] and the distance between the superimposed C $\beta$ 's.

The formula is:

$\mathrm{C} \beta-\mathrm{S}=\mathrm{TF} \times\left(\frac{D}{-6}+1\right)$

$\mathrm{C} \beta-\mathrm{S}$ is the score for each $\mathrm{C} \beta$, TF the factor from Tudos amino acid substitution matrix for isomorphic replacement,

Table 1

After five selection cycles using a phage library of cyclic constrained $7 \mathrm{mer}$ peptides and polyclonal affinity chromatography-purified anti-mHSP65 antibodies from subjects with sonographically proven carotid atherosclerosis 22 phage clones were isolated

\begin{tabular}{|c|c|c|c|}
\hline No. & $\begin{array}{l}\text { Amino acid sequences of } \\
\text { random peptide inserts }\end{array}$ & $\begin{array}{l}\mathrm{OD}_{(410)} \text { in } \\
\text { ELISA }\end{array}$ & $\begin{array}{l}\text { Frequency of } \\
\text { selection }\end{array}$ \\
\hline 1 & C I G S P S T N C & 735 & $14 x$ \\
\hline 2 & C I G S P S T N C & 701 & $14 \times$ \\
\hline 3 & C I GSPSTNC & 689 & $14 \times$ \\
\hline 4 & C S F H Y Q N R C & 682 & $3 x$ \\
\hline 5 & C I G S P S T N C & 681 & $14 \times$ \\
\hline 6 & C I G S P S T N C & 675 & $14 \times$ \\
\hline 7 & C S F H Y Q N R C & 672 & $3 \times$ \\
\hline 8 & C R Q S R K R P C & 670 & $1 \times$ \\
\hline 9 & C I G S P S T N C & 668 & $14 \times$ \\
\hline 10 & C I G S P S T N C & 665 & $14 \times$ \\
\hline 11 & C I G S P S T N C & 660 & $14 \times$ \\
\hline 12 & C S R T S S R T C & 657 & $1 \times$ \\
\hline 13 & C I G S P S T N C & 652 & $14 \times$ \\
\hline 14 & C S F H Y Q N R C & 648 & $3 \times$ \\
\hline 15 & C E L S P S S R C & 648 & $1 \times$ \\
\hline 16 & C T R W A G R P C & 639 & $1 \times$ \\
\hline 17 & C I G S P S T N C & 638 & $14 \times$ \\
\hline 18 & C L K R S K L R C & 630 & $1 \times$ \\
\hline 19 & C I G S P S T N C & 622 & $14 \times$ \\
\hline 20 & C I G S P S T N C & 622 & $14 \times$ \\
\hline 21 & C I G S P S T N C & 615 & $14 \times$ \\
\hline 22 & C I G S P S T N C & 608 & $14 \times$ \\
\hline - & CPES H I N Q C & $119^{\mathrm{a}}$ & - \\
\hline
\end{tabular}

The clones were tested in ELISA for binding affinity to the selection antibody and sequenced to determine their binding motifs. One binding motif was found 14 times (CIGSPSTNC), one three times (CSFHYQNRC) among the 22 phage clones sequenced. As negative control a non-selected and nonbinding clone was also sequenced (CPESHINQC).

${ }^{a}$ Negative control. and $D$ is the distance between superimposed C $\beta$ 's ( $\AA$ ) (if distance $>6 \AA$ then distance $=6 \AA$ ).

By summing the scores obtained for each $\mathrm{C} \beta$ an alignment score is calculated for every superimposition (120 superimpositions for each $C \beta$ ). Finally, the amino acids of the superimpositions evoking the highest alignment scores are recorded. (The program was written in MATLAB 6.0. The source code is available on request from the authors.)

\section{Results}

Five phage display selection rounds were performed with the polyclonal human anti-mHSP65 antibody. Before the last two selection rounds, negative selection with a polyclonal nonsense antibody was performed. Finally, 50 phage clones were isolated and tested in ELISA for their binding to the antibody, they were selected for. Thirty-seven of 50 clones (74\%) showed a positive reaction in ELISA $\left(\mathrm{OD}_{(410)}>400\right)$, versus 0 of 10 clones selected as negative controls $\left(\mathrm{OD}_{(410)}<150\right)$. DNA from the 22 strongest binding clones $\left(\mathrm{OD}_{(410)}>600\right)$ was isolated and their random peptide inserts sequenced
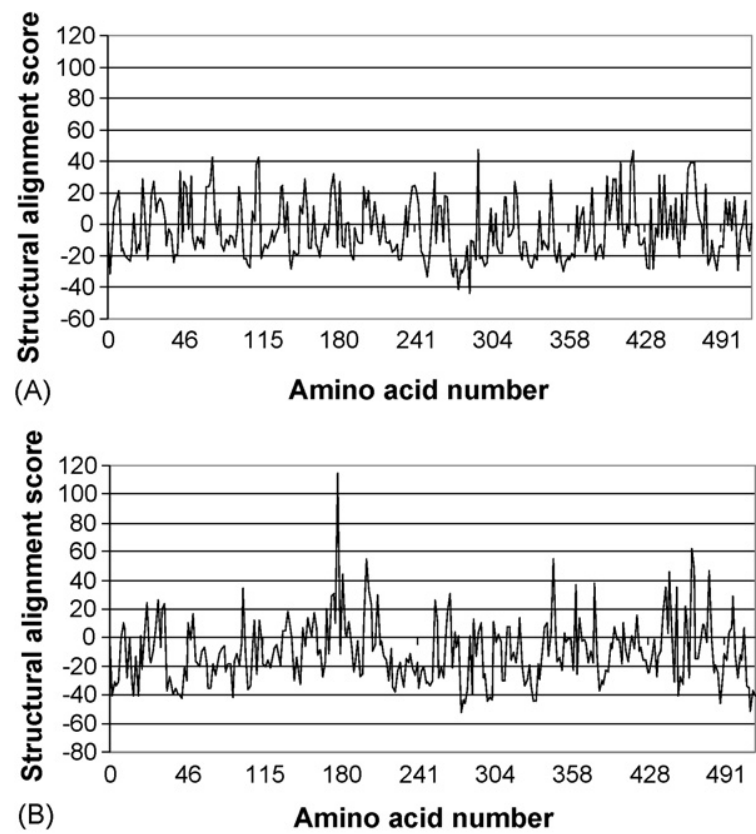

Fig. 1. (A) Negative control: structural alignment of substructure 3-7 (amino acids ESHIN) of not positively selected cyclic random peptide CPESHINQC onto the surface of mHSP65. The calculated structural alignment score of each surface exposed amino acid is plotted. It can be seen, that the "noise" of the algorithm is about 45 , therefore a hit was considered positive only if the alignment score exceeded the value of 90. (B) Structural alignment of substructure 3-7 (amino acids FHYQN) of positively selected cyclic peptide CSFHYQNRC onto the surface of mHSP65. A structural alignment score above 110 is observed at amino acid 178 , suggesting that the threedimensional coordinates of the five C $\beta$ 's of amino acids FHYQN match structurally to C $\beta$ 's of amino acid 178 and its 4 spatially nearest amino acids and that the types of the amino acids involved correspond according to the amino acid substitution matrix of Tudos et al. [30]. 
(Table 1). Interestingly, one random peptide was selected 14 times (CIGSPSTNC), one 3 times (CSFHYQNRC), and 5 motifs appeared only once among the 22 sequenced phage clones. Additionally, one non-binding clone was sequenced as negative control.

The structures of cyclic peptide motifs that were repeatedly selected (CIGSPSTNC, CSFHYQNRC), and the structure of a negative control peptide (CPESHINQC) were generated in silico by energy minimization of a starting structure in a $12 \AA$ water-box and subsequent simulated annealing. For each peptide, three different energy minima, corresponding to three different structures, were calculated. The structures of mHSP65 and hHSP60 were obtained from homology modelling and subsequent refinement in a force field. For the structural alignment calculations, only the surface-accessible amino acids from the protein were used.
Optimal superimpositions of sets of five adjacent C $\beta$ 's from the protein surface with the structures of the cyclic peptide motifs were generated by a newly developed program for structural alignment. Thus, to compare five $C \beta$ 's from the protein with five $C \beta$ 's from the cyclic peptide, the structures of the cyclic peptides (nine amino acids each, each peptide having a cysteine on positions 1 and 9) were divided into three substructures each (from amino acids 2-6, 3-7 and 4-8, respectively). Structural alignment scores were calculated for the three substructures of each cyclic peptide. None of the substructures from the negative control peptide CPESHINQC produced significant hits on mHSP65 or hHSP60. From these experiments, the highest "noise" value was estimated to be about 45 (Fig. 1A). In the following experiments, only scores higher than 90 were considered to be a positive hit. A strong positive hit for CSFHYQNRC

Table 2

The peptide structures of the binding motifs selected several times (CIGSPSTNC and CSFHYQNRC) were generated in silico by simulated annealing

\begin{tabular}{|c|c|c|c|}
\hline Peptide CIGSPSTNC & Substructure 2-6 (IGSPS) & Substructure 3-7 (GSPST) & Substructure 4-8 (SPSTN) \\
\hline \multicolumn{4}{|l|}{ mHSP65 } \\
\hline \multicolumn{4}{|l|}{1 st hit } \\
\hline Score & 85 & 116 & 75 \\
\hline Involved amino acids & $238,239,241,242,243$ & $31,32,33,477,478$ & $252,253,254,257,258$ \\
\hline \multicolumn{4}{|l|}{ 2nd hit } \\
\hline Score & 71 & 93 & 69 \\
\hline Involved amino acids & $151,152,153,154,392$ & $40,43,44,45,46$ & $3,63,519,520,521$ \\
\hline \multicolumn{4}{|l|}{ hHSP60 } \\
\hline \multicolumn{4}{|l|}{ 1st hit } \\
\hline Score & 92 & 108 & 88 \\
\hline Involved amino acids & $203,206,207,208,210$ & $205,206,208,210,325$ & $139,140,143,147,158$ \\
\hline \multicolumn{4}{|l|}{ 2nd hit } \\
\hline Score & 90 & 101 & 85 \\
\hline Involved amino acids & $224,226,227,228,253$ & $40,43,44,45,386$ & $53,56,57,73,77$ \\
\hline Peptide CSFHYQNRC & Substructure 2-6 (SFHYQ) & Substructure 3-7 (FHYQN) & Substructure 4-8 (CIGSPSTNC) \\
\hline \multicolumn{4}{|l|}{ mHSP65 } \\
\hline \multicolumn{4}{|l|}{ 1st hit } \\
\hline Score & 58 & 115 & 61 \\
\hline Involved amino acids & $82,123,497,501,505$ & $178,179,180,181,183$ & $357,359,360,361,363$ \\
\hline \multicolumn{4}{|l|}{ 2nd hit } \\
\hline Score & 56 & 62 & 60 \\
\hline Involved amino acids & $287,296,297,299,313$ & $464,467,468,469,472$ & $202,207,208,323,324$ \\
\hline \multicolumn{4}{|l|}{ hHSP60 } \\
\hline \multicolumn{4}{|l|}{ 1st hit } \\
\hline Score & 71 & 64 & 85 \\
\hline Involved amino acids & $467,470,471,472,475$ & $237,297,312,314,315$ & $80,82,396,400,403$ \\
\hline \multicolumn{4}{|l|}{ 2nd hit } \\
\hline Score & 65 & 52 & 53 \\
\hline Involved amino acids & $37,39,40,44,45$ & $40,43,44,45,386$ & $71,73,74,77,78$ \\
\hline
\end{tabular}

For every peptide, three structures were generated. The structures were aligned structurally onto the surface of mHSP65 and hHSP60, whereas no significant difference between the three different structures of each peptide was observed, therefore consequently the mean values of all three structures are presented. Since the alignment algorithm is based on 5mer peptides, the structures were divided into three substructures: amino acid 2-6, 3-7 and 4-8, respectively. For CIGSPSTNC, two positive hits (score > 90) were observed for substructure 3-7 at different sites for mHSP65 as well as for hHSP60. For CSFHYQNRC a strong hit was observed for substructure 3-7 on mHSP65, and no hit on hHSP60. 
(substructure 3-7) on mHSP65 is shown in Fig. 1B. Peptide CSFHYQNRC failed to show a strong hit on hHSP60. For CIGSPSTNC (substructure 3-7), two strong hits on mHSP65 as well as on hHSP60 were observed (Table 2). There were no significant differences with respect to the alignment scores among the three simulated structures of each cyclic peptide (data not shown). Thus, the scores reflect the mean values from the different structures.

Optimal all-atom-superimpositions of the cyclic peptide structures with the identified amino acids from the protein surface were generated with the fit algorithm implemented in the Swiss pdb-viewer [23] and adjusted manually. Superimposition of substructure 3-7 of the peptide CSFHYQNRC with the amino acids Asn 178, Thr 179, Phe 180, Gly 181, Gln 183 from mHSP65 showed in seven positions homology with respect to amino acid sidechains orientation of carboxy- and amide groups from the backbone (Fig. 2A). The surface exposition of this epitope is shown in Fig. 2B. For peptide CIGSPSTNC (substructure 3-7), reasonable superimpositions were possible only with the "second-strongest" hits, e.g. Lys 40, Gly 43, Ala 44, Pro 45, Thr 46 of mHSP65 (consistency in six positions) and, albeit to a lower extent, with Gln 40, Gly 43, Ser 44, Pro 45, Val 386 of hHSP60 (consistency in five positions) (Fig. 3AB). No meaningful overlay was possible for mHSP65 Pro 31, Ser 32, Gly 33, Gln 477, Thr 478 and hHSP60 Thr 205, Ser 206, Gly 208, Lys 210, Thr 325 (Fig. 3C and D), although these amino acids showed the strongest hits in the $\mathrm{C} \beta$-based structural alignment.

\section{Discussion}

Autoimmunity to human HSP60, triggered by crossreactivity of the protective immune response directed against microbial HSP60's or by a bona fide autoimmune reaction against biochemically altered autologous HSP60, is believed to be involved in many autoimmune diseases, including atherosclerosis [31]. Purified human anti-bacterial HSP60 antibodies showed cytotoxicity on stressed, but not unstressed, human endothelial cells [19,32]. We recently reported linear (continuous) B-cell epitopes shared between microbial and human HSP60 [16]. However, in contrast to $\mathrm{T}$ cells, antibodies generally recognize three-dimensional structural motifs that are not necessarily made up of a single linear peptide sequence. Mapping only linear (continuous) epitopes by means of peptides derived from the primary amino acid sequence does not, therefore, necessarily lead to identification of all epitopes relevant to pathogenesis of atherosclerosis.

We purified polyclonal human anti-mHSP65 antibodies from clinically healthy subjects with sonographically proven carotid atherosclerosis by affinity chromatography. A special phage-display peptide library was used to identify
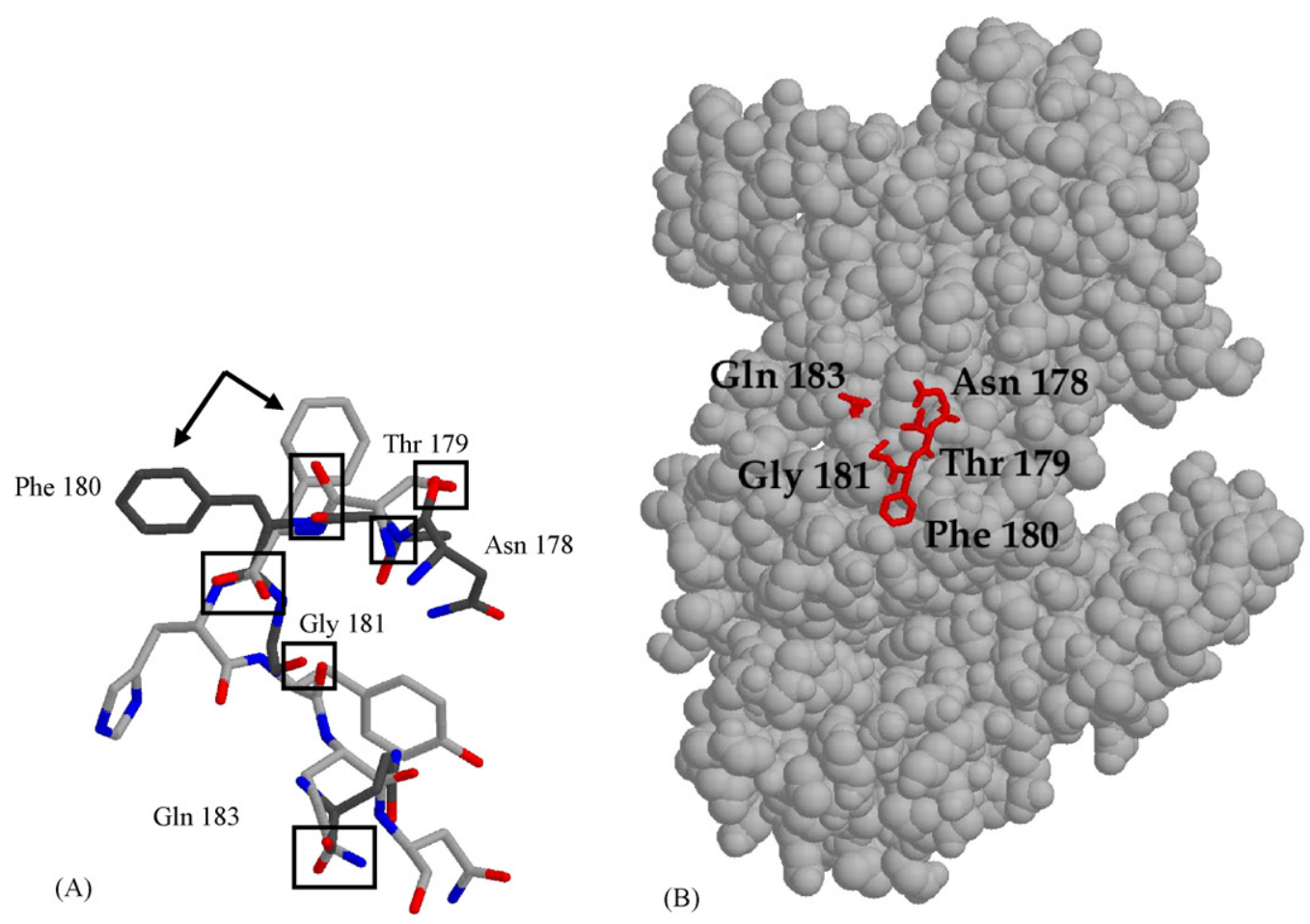

Fig. 2. (A) Optimal structural all-atom superimposition of peptide CSFHYQNRC substructure 3-7 (amino acids FHYQN, light grey) with the identified epitope of mHSP65 (dark grey). An obvious congruence of functional groups can be seen (matching regions are marked with boxes and arrows). Only mHSP65 amino acids participating in the epitope are shown and labelled. The superimposition was computed using the Fit-algorithm implemented in the SwissPdB Viewer [23]. (B) Surface localization of the identified epitope (red) on the structure of mHSP65. 

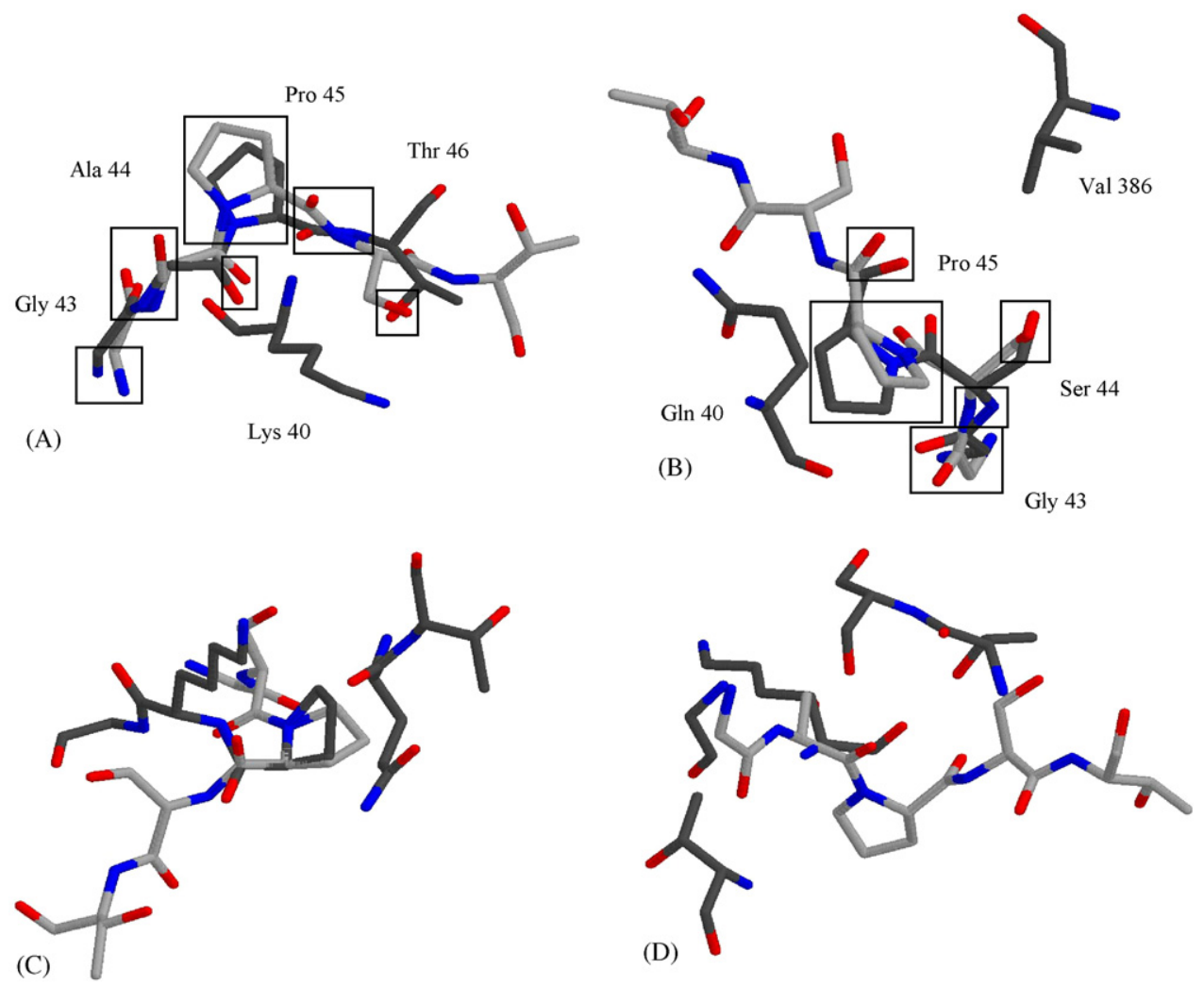

Fig. 3. Optimal structural superimpositions of peptide CIGSPSTNC substructure 3-7 (amino acids GSPST, light grey) with the identified epitope regions of mHSP65 and hHSP60 (dark grey). Matching regions are marked with boxes. The peptide structure could be meaningfully overlaid with mHSP65 amino acids Lys 40, Gly 43, Ala 44, Pro 45, Thr 46 (A) and hHSP60 Gln 40, Gly 43, Ser 44, Pro 45, Val 386 (B). However, reasonable superimpositions were not possible for the two epitope regions which were suggested also from the structural alignment algorithm (Table 2): mHSP65: Pro 31, Ser 32, Gly 33, Gln 477, Thr 478 (C) and hHSP60: Thr 205, Ser 206, Gly 208, Lys 210, Thr 325 (D). This example shows, that strong hits have to be verified by all-atom superimpositions to exclude false positive results. Superimpositions were computed using the Fit-algorithm implemented in the SwissPdB Viewer [23].

the conformational epitopes recognized by these antibodies. In this type of library, the surface of every phage clone bears repeated motifs of seven random amino acids flanked by two cysteines each, resulting in a constrained ring-shaped structure with low flexibility of the binding motif. Finally, 22 phage clones binding strongly to the affinity chromatography-purified anti-mHSP65 antibodies were isolated and sequenced to determine their binding motifs. Interestingly, one binding motif was selected 14 times (CIGSPSTNC), one 3 times (CSFHYQNRC) and five motifs appeared once among the 22 sequenced phage clones.

The three-dimensional structures of the repeatedly selected binding motifs were simulated in silico. From the view of molecular modelling, cyclic 9mer peptides have the advantage of a much more limited number of reasonable structures than, for example, linear 9 mer peptides. The structures of hHSP60 and mHSP65 have not been determined experimentally yet, but several X-ray structures of highly homologue proteins from the same class are available. Therefore it was possible to obtain reliable structures of hHSP60 and mHSP 65 by homology modelling.

No adequate method for correct assignment of the 9mer cyclic peptide structures to the surface of the HSP60 protein could be found. Several published studies for conformational epitope mapping aligned linear peptides onto a protein surface [33-35], however, a reasonable method using both, structural information of the protein and of the phage display derived peptide motif, is still not available. Existing programs for aligning structures did not prove to be sufficient for our purposes, being either designed to compare small molecules to identify pharmacophores (e.g. CoMFA or DISCO) or to align large numbers of protein backbone structures in fold recognition or function prediction studies (e.g. STAMP).

Therefore we decided to develop a new alignment algorithm based on the $C \beta$ atoms of the amino acids. The $C \beta$ atom of an amino acid is the first atom that contains information about the structural orientation of the sidechain, but the rigid anchoring of the $\mathrm{C} \alpha$ atom in the protein backbone limits the spatial flexibility of the $C \beta$ atom. Since antibody binding is possible on the protein surface only the alignment was limited to $C \beta s$ of surface-exposed amino acids of mHSP65 and hHSP60.

Sets of five spatial adjacent $C \beta$ s (for glycines $C \alpha$ atoms) were used in the structural alignment procedure for two reasons: (i) the core region of an epitope is usually made up of five to six amino acids, and we did not want to increase the 

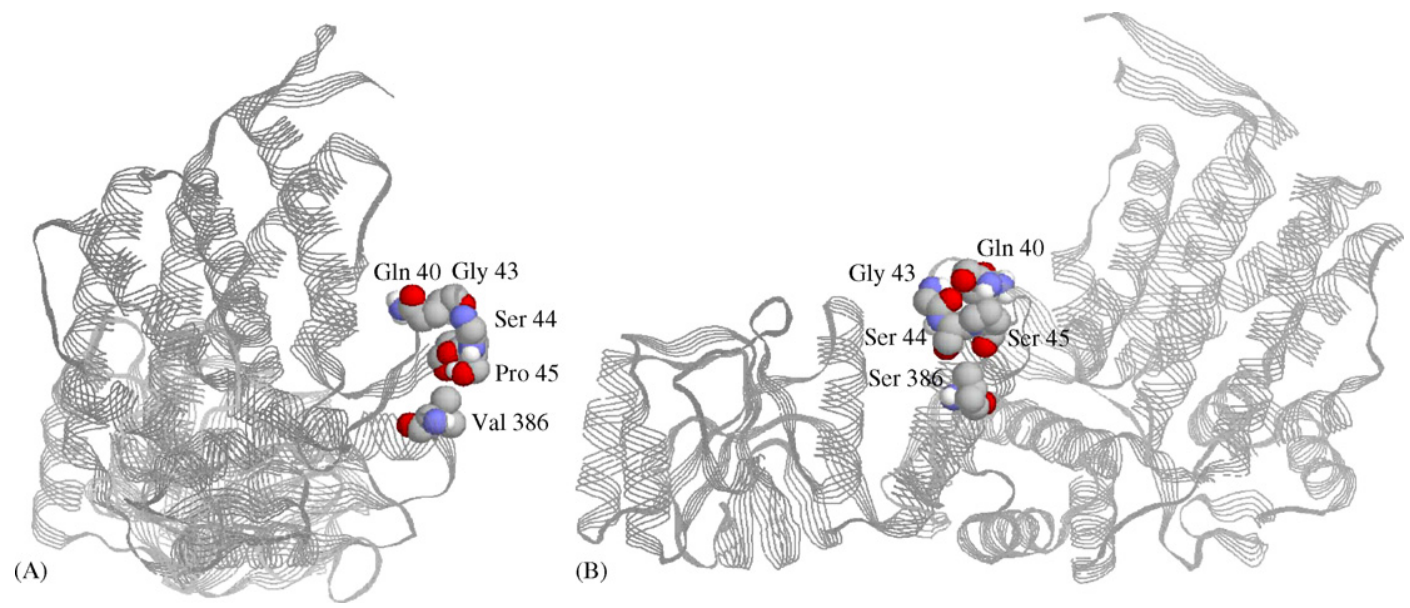

Fig. 4. (A) Front and (B) side views of the modelled structure of hHSP60. Amino acids of the putative cross-reactive conformational epitope are highlighted. The epitope lies in a highly exposed loop that should be predisposed as antibody recognition site. However, it was not identified as an epitope by any of the previous studies focussing primarily on linear epitopes.

risk of false positive hits by performing the algorithm on too many amino acids. (ii) Obtaining the best structural superimposition requires computation of all possible permutations. Thus, five $C \beta$ s require 120 operations (=5!), and encompassing seven amino acids increases this number to $5020(=7$ !), making this method impractical.

We generated optimal structural superimpositions of groups of five adjacent $C \beta$ atoms from the protein surfaceexposed amino acids with the $\mathrm{C} \beta$ atoms from the cyclic peptide structure. For each superimposition, an alignment score was calculated based on the congruence of the amino acids, expressed according to the amino acid substitution matrix for isomorphic replacement by Tudos et al. [30] and weighed by the quality of the superimposition, expressed as distance between protein and peptide $C \beta s$.

The "noise" generated by unspecific hits was estimated by running the method with a non-binding peptide (Fig. 1A). Peptide CSFHYQNRC, which was present three times among the sequenced clones, revealed that substructure 3-7 (corresponding to amino acids $\mathrm{HYQN}$ ) is related to a region around amino acid 179 from mHSP65. All-atom-superimposition plots confirmed this result (Fig. 2A). No significant hit with $\mathrm{C} \beta$ s from the surface of hHSP60 was observed, suggesting that this epitope is not cross-reactive between bacterial and human HSP60's.

Fourteen of 22 sequenced clones contained the motif CIGSPSTNC. Structural alignment of substructure 3-7 with mHSP65 and hHSP60 revealed two hits on each protein. However, superimpositions of the peptide structure with the four identified epitope regions using all backbone and sidechain atoms revealed that two epitopes, mHSP65 (Lys 40, Gly 43, Ala 44, Pro 45, Thr 46) and its homologue hHSP60 (Gln 40, Gly 43, Ser 44, Pro 45, Val 386), could be overlaid plausibly (Fig. 3A and B), while reasonable allatom-superimpositions were not possible for the other two epitopes (Fig. 3C and D).
This example indicates the need for caution in interpreting the results of our structural alignment method, and verification by all-atom-superimposition plots should be performed to exclude false hits even if matching $\mathrm{C} \beta$ coordinates and amino acid types suggest a strong positive hit.

In this study, the epitopes were identified using antibodies purified from subjects with proven carotid atherosclerosis. Especially the fact that one epitope motif was found in both human and mycobacterial HSP60 structures makes this epitope a promising candidate for further investigations on cross-reactive epitopes. In the three-dimensional structure of the protein the epitope is located in a very surface-exposed loop that is predisposed to be a strong antigenic area (Fig. 4).

It is known, that HSP60, or at least parts of it, may be exposed on the cell surface [4-6]. Consequently, the next steps are to confirm the presence of this epitope within early atherosclerotic lesions and whether immune reactions against this epitope are involved in pathogenesis of atherosclerosis.

\section{Acknowledgements}

The work was supported by the Austrian Research Fund (project 14741-MED), a grant from MEDAC GmbH, Hamburg, Germany and the European Vascular Genomics Network - EVGN (LSHM-CT-2003-S03254).

\section{References}

[1] Ross R. Atherosclerosis - an inflammatory disease. N Engl J Med 1999;340:115-26.

[2] Karlin S, Brocchieri L. Heat shock protein 60 sequence comparisons: duplications, lateral transfer, and mitochondrial evolution. Proc Natl Acad Sci USA 2000;97:11348-53.

[3] Zugel U, Kaufmann SH. Role of heat shock proteins in protection from and pathogenesis of infectious diseases. Clin Microbiol Rev 1999;12:19-39. 
[4] Wand-Wurttenberger A, Schoel B, Ivanyi J, Kaufmann SH. Surface expression by mononuclear phagocytes of an epitope shared with mycobacterial heat shock protein 60. Eur J Immunol 1991;21:1089-92.

[5] Xu Q, Schett G, Seitz CS, Hu Y, Gupta RS, Wick G. Surface staining and cytotoxic activity of heat-shock protein 60 antibody in stressed aortic endothelial cells. Circ Res 1994;75:1078-85.

[6] Soltys BJ, Gupta RS. Cell surface localization of the $60 \mathrm{kDa}$ heat shock chaperonin protein (hsp60) in mammalian cells. Cell Biol Int 1997;21:315-20.

[7] Danesh J, Collins R, Peto R. Chronic infections and coronary heart disease: is there a link? Lancet 1997;350:430-6.

[8] Mayr M, Kiechl S, Willeit J, Wick G, Xu Q. Infections, immunity, and atherosclerosis: associations of antibodies to Chlamydia pneumoniae, Helicobacter pylori, and cytomegalovirus with immune reactions to heat-shock protein 60 and carotid or femoral atherosclerosis. Circulation 2000;102:833-9.

[9] Kiechl S, Egger G, Mayr M, et al. Chronic infections and the risk of carotid atherosclerosis: prospective results from a large population study. Circulation 2001;103:1064-70.

[10] Xu Q, Willeit J, Marosi M, et al. Association of serum antibodies to heatshock protein 65 with carotid atherosclerosis. Lancet 1993;341:255-9.

[11] Xu Q, Kiechl S, Mayr M, et al. Association of serum antibodies to heat-shock protein 65 with carotid atherosclerosis: clinical significance determined in a follow-up study. Circulation 1999;100:116974.

[12] Frostegard J, Lemne C, Andersson B, et al. Association of serum antibodies to heat-shock protein 65 with borderline hypertension. Hypertension 1997;29:40-4.

[13] Birnie DH, Holme ER, McKay IC, et al. Association between antibodies to heat shock protein 65 and coronary atherosclerosis. Possible mechanism of action of Helicobacter pylori and other bacterial infections in increasing cardiovascular risk. Eur Heart J 1998;19:38794.

[14] Burian K, Kis Z, Virok D, et al. Independent and joint effects of antibodies to human heat-shock protein 60 and Chlamydia pneumoniae infection in the development of coronary atherosclerosis. Circulation 2001;103:1503-8.

[15] Zhu J, Quyyumi AA, Rott D, et al. Antibodies to human heat-shock protein 60 are associated with the presence and severity of coronary artery disease: evidence for an autoimmune component of atherogenesis. Circulation 2001;103:1071-5.

[16] Perschinka H, Mayr M, Millonig G, et al. Cross-reactive B-cell epitopes of microbial and human heat shock protein 60/65 in atherosclerosis. Arterioscler Thromb Vasc Biol 2003;23:1060-5.

[17] Luzzago A, Felici F, Tramontano A, Pessi A, Cortese R. Mimicking of discontinuous epitopes by phage-displayed peptides. I. Epitope mapping of human $\mathrm{H}$ ferritin using a phage library of constrained peptides. Gene 1993;128:51-7.
[18] Willeit J, Kiechl S. Prevalence and risk factors of asymptomatic extracranial carotid artery atherosclerosis. A population-based study. Arterioscler Thromb 1993;13:661-8.

[19] Schett G, Xu Q, Amberger A, et al. Autoantibodies against heat shock protein 60 mediate endothelial cytotoxicity. J Clin Invest 1995;96:2569-77.

[20] Devlin JJ, Panganiban LC, Devlin PE. Random peptide libraries: a source of specific protein binding molecules. Science 1990;249:404-6.

[21] Case D, Pearlman D, Caldwell J, Cheatham I, Ross W, AMBER 6 San Francisco: University of California, 1999.

[22] Cornell W, Cieplak P, Bayly C, Gould I, Merz J. A second generation force field for the simulation of proteins, nucleic acids and organic molecules. J Am Chem Soc 1995;117:5179-97.

[23] Guex N, Peitsch MC. SWISS-MODEL and the Swiss-PdbViewer: an environment for comparative protein modeling. Electrophoresis 1997; 18:2714-23.

[24] Sanchez R, Pieper U, Melo F, et al. Protein structure modeling for structural genomics. Nat Struct Biol 2000;7(Suppl):986-90.

[25] Sali A, Kuriyan J. Challenges at the frontiers of structural biology. Trends Cell Biol 1999;9:M20-4.

[26] Van Gunsteren W, Billeter S, Eising A, Hünenberger P, Bimolecular simulation: the GROMOS96 manual and user guide Zürich: Hochschulverlag an der ETH, 1996.

[27] Fraczkiewicz R, Braun W. Exact and efficient analytical calculation of the accessible surface areas and their gradients for macromolecules. J Comp Chem 1998;19:319-33.

[28] Kabsch W. A solution for the best rotation to relate two sets of vectors. Acta Cryst A 1976;32:922-3.

[29] Kabsch W. A discussion of the solution for the best rotation to relate two sets of vectors. Acta Cryst A 1978;34:827-8.

[30] Tudos E, Cserzo M, Simon I. Predicting isomorphic residue replacements for protein design. Int J Pept Protein Res 1990;36:236-9.

[31] Wick G, Knoflach M, Xu Q. Autoimmune and inflammatory mechanisms in atherosclerosis. Annu Rev Immunol 2004;22:361-403.

[32] Mayr M, Metzler B, Kiechl S, et al. Endothelial cytotoxicity mediated by serum antibodies to heat shock proteins of Escherichia coli and Chlamydia pneumoniae: immune reactions to heat shock proteins as a possible link between infection and atherosclerosis. Circulation 1999;99:1560-6.

[33] Ganglberger E, Grunberger K, Sponer B, et al. Allergen mimotopes for 3-dimensional epitope search and induction of antibodies inhibiting human IgE. FASEB J 2000;14:2177-84.

[34] Moreau V, Granier C, Villard S, Laune D, Molina F. Discontinuous epitope prediction based on mimotope analysis. Bioinformatics 2006;22(9):1088-95. May 1.

[35] Schreiber A, Humbert M, Benz A, Dietrich U. 3D-Epitope-explorer (3DEX): localization of conformational epitopes within the threedimensional structures of proteins. J Comput Chem 2005;26:879-87. 